\title{
Trabajando la educación ambiental desde la metodología aprendizaje-servicio
}

DOI: $10.46932 / \mathrm{sfjdv} 2 \mathrm{n} 1-010$

Received in: November 1st, 2020

Accepted in: December 30th, 2020

\author{
José Santiago Álvarez-Muñoz \\ Dr. En Educación \\ Universidad de Murcia \\ E-mail: josesantiago.alvarez@um.es \\ Abraham Bernárdez-Gómez \\ Contratado Predoctoral FPI. Ref: BES-2017-081040 \\ Universidad de Murcia \\ Email: Abraham.bernandez@um.es \\ María Luisa Belmonte \\ Dra. en Investigación e innovación educativa \\ Universidad de Murcia \\ E-mail: marialuisa.belmonte@um.es
}

\section{RESUMEN}

La educación ambiental queda representada como uno de los ejes transversales que mayor protagonismo ocupa dentro del sistema educativo, cada vez más necesario ante la multitud de amenazas que atentan contra el desarrollo sostenible de nuestro mundo, encontrando en la educación la herramienta perfecta para formar a nuevas generaciones conscientes al respecto. No obstante, a fin de superar métodos de trabajo ya repetitivos y conservadores, se toma en consideración nuevas metodologías emergentes como el aprendizaje servicio, que aúna aprendizaje curricular y moral. Siguiente este marco, se expone un proyecto de aprendizaje servicio basado en el medio ambiente y la sostenibilidad desarrollado en el primer tramo de la Etapa de Educación Primaria. Un modelo de intervención en el que el alumnado es el protagonista y se desarrolla con una finalidad que se materializa, adquiriendo el aprendizaje desde la praxis directa en un contexto real.

Palabras clave: educación ambiental, educación primaria, aprendizaje-servicio, sostenibilidad.

\section{INTRODUCCIÓN}

\subsection{LA EDUCACIÓN AMBIENTAL: GENERANDO EL CAMBIO.}

Tal y como señala González Gaudiano (1997), la educación ambiental aparece por primera vez tras la celebración de la conferencia de educación ambiental de Tbilisi (Georgia) celebrada en el año 1977 por medio de la cual se exponen las bases de este eje transversal: comprensión de los elementos naturales que han influenciado sobre las formas de vida del ser humano, conocimiento de las repercusiones que comporta la acción del ser humano en nuestro entorno y, por último, un principio que ahonda sobre el ámbito actitudinal, es decir, el ejercicio de una actitud crítica frente a la acción entrópica y las 
consecuencias que éstas suponen. Inicialmente partía de una definición demasiado conceptual que, conforme pasaban los años, se le fue asociando acotaciones de índole social hasta ampliar el rango conceptual contemplado la convergencia de elementos de carácter socioeconómico, ecológico y cultural (Novo, 1998).

En este sentido, la educación ambiental se ve representado como un fenómeno global que acota la educación formal a través de su incorporación en el currículo oficial y oculto de los sistemas educativos, es decir, la escuela, instituto o universidad. Sin embargo, este tipo de contenidos, deben ir más allá de lo meramente formal atendiendo de forma coordinada con medios educativos informales de gran relevancia como los medios de comunicación o la propia familia, siendo esta la fórmula idónea para poder llegar al fin último: la concienciación social generalizada Así, ambas dimensiones educativas se han de conjugan en pro de logar una transformación de las conductas desde un ámbito más planificado junto a otro que surge desde un entorno natural y familiar (Belmonte y Bernárdez-Gómez, 2020; Calixto, 2008; Trillo, Parada y Bernárdez-Gómez, 2020).

Tal es la amplitud del tema que nos lleva a su inmersión dentro de la cultura popular pues este tema ha de ser asumido desde nuestro proceder como ser humano. Para tal fin se ha de perfilar la educación ambiental como un elemento de relevancia dentro de la humanidad que se ve contemplado en diferentes parcelas de conocimiento de la humanidad como la agricultura o la industria, además de la educación que ha sido mencionada anteriormente. De esta forma, emerge la cultura ambiental fruto de las diferentes acciones entrópicas desarrolladas en nuestro entorno la cual, además de un enfoque educativo, se ha de abordar desde una perspectiva cultural, que posibilite la asimilación de una serie de estilos, hábitos, costumbres...que definen nuestras formas de vida, hallando en el aprendizaje medioambiental la mejor herramienta para tal fin (Alonso, Nuñez y Hernández, 2015).

Por consiguiente, surge la educación ambiental como recurso para alcanzar una solución a esta situación que solucione el presente y dé un mejor futuro ante el cumulo de errores cometidos a lo largo de la historia. Se establece como la vía para la adquisición de las nociones teóricas, prácticas y actitudinales necesarios para la construcción de una cultura ambiental que supere los preceptos teóricos hasta materializarse en acciones concretas (Bernárdez-Gómez y Belmonte, 2020a, 2020b). La escuela, junto a otros agentes, se considera uno de los medios más idóneos, ejerciendo su acción por medio de actividades transversales o grandes proyectos anules. A continuación, se van a mostrar las posibilidades de la educación ambiental dentro del contexto escolar como medio para cimentar las bases que posibilitará aprendizajes posteriores (Novo, 1995).

La educación se configura como una de las armas sociales que posibilita el cambio dado que se gestiona de manera que ayuda a generar el cambio en pro de ocasionar mejoras que hacen de nuestro 
entorno un medio facilitador para la vida. Dentro del ámbito educativo, más concretamente el formal, se póstula la escuela como una de las instituciones más importantes dado que sitúa a la población que tiene un rango de edad más importante de cara a la adquisición de hábitos, normas, actitudes... momento vital en el que se encuentran sensible para la asimilación de este tipo de aprendizajes, construyendo la base de lo que definirá nuestras acciones del futuro como ciudadanos de esta sociedad democrática del siglo XXI.

La formación cívica y humana en varias ocasiones queda excluido de los currículos oficiales que definen los aprendizajes mínimos, no obstante, también se ha de contemplar un currículo oculto que hace del conocimiento un saber que no es cerrado. Éstos contenidos que no se ubican de forma concreta en una materia se suelen abordar de diversas maneras, una de ellas es la transversalidad que se define como el tratamiento de un contenido de forma simultánea desde varias áreas curriculares a la misma vez. El equipo docente, desde un enfoque comunitario, coordinador y cooperativo, deberá aunar fuerzas para preestablecer las diversas acciones que se van a desarrollar a lo largo del curso escolar desde las diversas materias curriculares.

Muchos de los problemas respecto al tratamiento de la educación ambiental radican en la escasa predisposición de los docentes para su inclusión en el proceder docente, la poca formación respecto al tema y la dificultad de ajustar este tipo de acciones en las programaciones. No obstante, cada vez se ha de ser más consciente sobre su obligatoriedad dentro de las estructuras escolares de trabajo dado que son temas protagonistas dentro de nuestra sociedad los cuales ayudan a enriquecer el proceso de educación integral de los menores (Pardo, 1995).

De esta forma, dentro del entorno escolar, la educación ambiental surge de la vivencia directa ante una crisis ecológica que demanda de nuevas formas de relación entre el ser humano y el entorno, requiriendo de una visión pedagógica para el ofrecimiento de experiencias escolares diversas que engloben el campo de conocimiento que supone este eje educacional transversal. No obstante, dada su relevancia y "relativa" juventud, requiere de un enfoque pedagógico diferente que diste de los modelos conservadores y tradicionales, ofreciendo un marco educativo que contemple la transversalidad, los principios éticos, la innovación conceptual y la innovación metodológica para conseguir cambio de actitudes y comportamientos. Todo ello conceptualizado dentro de un nuevo estilo educativo que requiere de un sistema abierto y flexible, fluctuaciones adecuadas, comprensión al cambio y estrategias de innovación.

\subsection{LA METODOLOGÍA APRENDIZAJE POR SERVICIO: APRENDE ACTUANDO.}

Según señala Puig y Palos (2006), dicha metodología sustenta el éxito de aprendizaje en la ayuda mutua, haciendo más importante conceptos como la cohesión, solidaridad o igualdad para el avance y 
progresión del individuo, elementos que se ven relacionados con la ayuda mutua desde tres planos de actuación: la cooperación entre los compañeros para dar un servicio a la comunidad, la acción de servicio hacia un grupo de población determinado con una necesidad existente y, por último, la responsabilidad social propia como el deber ciudadano. Una experiencia óptima para la educación a la ciudadanía que estimule la cultura democrática en una sociedad sensible a los cambios e inmersa en una crisis de valores.

Esta nueva forma de entender la enseñanza erradica la idea de evaluación por competencia, es decir, un modelo que entiende como única forma de calificación a través del trabajo individual, situación que ocasiona malestar y presión en el alumnado. Por el contrario, la inclusión del altruismo dentro del trabajo con agrupaciones colaborativas hace florecer una serie de beneficios: emociones positivas, autoestima, auto concepto...en conclusión, un ejercicio de autoayuda en el que se aporta del propio ser para recibir más de lo que se da.

Uno de los rasgos característicos que definen su éxito educativo es el carácter práctico que incorpora que permite acceder a los aprendizajes desde un contexto real, posibilitándose como una materialización de los aprendizajes que lo hace más accesibles. La práctica no sólo se quedaría en el mero ejercicio, sino que desde el ApS se da un paso más al abordar aprendizajes que incluyen la reflexión de la propia práctica para emitir un juicio u opinión propia fruto de la problemática planteado, pudiendo tomar conciencia de un amplio rango de temas desde la educación medio ambiental hasta la inclusión educativa.

Después de todo lo comentado cabe resaltar como una definición que lo sintetiza la marcada por Tapia (2008): una actividad o programa de servicio solidario protagonizado por los estudiantes, orientado a atender eficazmente las necesidades de una comunidad, y planificada de forma integrada con los contenidos curriculares con el objetivo de optimizar los aprendizajes (p.43). Una educación vivencial en el que el ser es el protagonista de la acción y el proceso, es decir, el aprendizaje y la enseñanza.

Para el entendimiento de la metodología ApS se hace necesario que se delimiten y conceptualicen bien los elementos clave que subyacen de ésta. Una de las piezas clave es el voluntariado el cual se entiende como una acción del ciudadano en pro de satisfacer una necesidad de un colectivo específico sin ninguna remuneración económica ante ello, lo único que recibe es la satisfacción personal, una de las principales motivaciones por la que se realiza esta acción (Vecina Jiménez, Chacón Fuertes, y Sueiro Abad, 2009).

Por lo cual, este servicio a la comunidad no ha de ser cualquiera, se debe acceder y abordar desde una iniciativa solidaria que ayude a satisfacer una necesidad contemplada dentro de un colectivo o grupo específico propio de nuestro entorno en favor de que, tras la intervención o trabajo de campo llevado a cabo, se dé un cambio positivo y de mejora. Acción que contribuye a que el alumnado entienda el modelo 
de participación ciudadana que se postula desde el voluntariado y el papel que éste desempeña en el avance de nuestra sociedad, otorgando una instrucción esencial para la escala de valores del ser.

Sin embargo, tampoco se puede generalizar que todo voluntariado regularizado es ApS puesto que ha de constar una connotación educativa para que se identifique con tal metodología. No todo voluntariado, aunque sea fructífero personalmente, lleva al aprendizaje, ha de presentar un servicio que se adhiere al aprendizaje práctico de una serie de contenidos incluidos en el currículo escolar y/o oculto, elementos que se han de aprender a través de la práctica y reflexión del servicio realizado. Por lo tanto, la incorporación de una dimensión didáctica al voluntariado permite el acceso al aprendizaje y forjar, por lo tanto, una experiencia ApS.

Desde la otra perspectiva, debemos remarcar que se requiere de un servicio a nuestra comunidad para que se presente esta metodología dentro de la intervención didáctica, es decir, no todo aprendizaje se objeta como experiencia ApS. Nuestra intervención docente ha de traspasar los muros y ser desencadenante positivo en nuestro entorno cercano, perfilándose como motor social hacia el cambio a través de la creación de experiencias que posibilite el acercamiento al objeto didáctico desde el servicio a nuestra comunidad (Campo, 2014).

\section{MÉTODO}

En el presente apartado se expone todos los parámetros metodológicos que definen la intervención de aprendizaje por servicio ideada para desarrollar en el primer tramo de Educación Primaria de cualquier centro educativo, queda identificado como el proyecto denominado como "Seres solidarios con el medio ambiente".

\subsection{OBJETIVOS}

A continuación, se presenta en la tabla 1 el objetivo general, referenciado como el propósito principal, junto los objetivos específicos que articulan y orientan de forma más concreta la intervención ejecutada. 
Tabla 1.

Relación de objetivos general y específicos

\section{Objetivo general}

Concienciar acerca del respeto y control del medio ambiente, actuando de forma responsable y

respetuosa en interacción con nuestro entorno vivido. Objetivos específicos

Concienciar sobre las problemáticas que ahondan en la interacción con nuestro medio natural.

Identificar las situaciones o elementos que generan contaminación en nuestro perjudicando las condiciones naturales del mismo.

Tomar un rol participativo y activo en la toma de decisiones e iniciativas en pro de la protección y cuidado del medio ambiente.

Redactar y elaborar un plan de trabajo para asegurar la creación de un espacio que contemple el reciclado y limpieza como premisas básicas

Demostrar una actitud participativa, respetuosa y activa ante las diferentes situaciones compartidas y cooperativas en pro del medio ambiente.

Impulsar el ámbito artístico y creativo en el diseño y preparación de materiales de diferente índole en relación al campo conceptual de la educación ecológica.

\subsection{TEMPORALIZACIÓN E INTERVENCIÓN}

A la hora de la puesta a cabo de toda la intervención configura un proceso de trabajo que se perfila dentro de un carácter sistemático y progresivo que posibilita la adhesión de los aprendizajes dentro de un programa en crecimiento que le otorga un sentido a la intervención desarrollada, facilitando la transmisión de los conocimientos. Éste se enmarcará dentro de tres grandes fases, una para cada trimestre, las cuales son:

- Sensibilización: En un primer contacto se va a tomar una conciencia acerca de los problemas medio ambientales que encontramos dentro de nuestro entorno partiendo de nuestras propias prácticas para darle una mayor significatividad. De esta forma, acercaremos al alumnado al objeto de aprendizaje desde una perspectiva personal haciendo comprender el calibre de la problemática que estamos abordando y, además, la relevancia que el rol que este desempeñe influye en el desarrollo de la situación protagonista dentro de nuestro panorama social actual.

- Acercándose a la teoría desde la práctica: A continuación, tocará el momento de partir de situaciones reales y prácticas las cuales estamos viviendo, pero no somos consciente de ello. Además, dicho tratamiento no se abordará desde la mera descripción, sino que éstos deberán analizar y transferir a situaciones prácticas todo lo estudiado de manera que se invite a la crítica y a la reflexión para que, a su vez, refuercen los hábitos y prácticas que el alumnado desarrolla en su tiempo libre, centrándonos en la importancia y relevancia del papel del ser humano como medio posibilitador para el cambio.

- Desarrollando acciones en nuestro entorno: Por último, a partir de lo abordado desde las actividades teóricas y prácticas, se desarrollará una serie de intervenciones que hagan a la educación como medio representativo para el cambio social. Tal premisa quiere decir que se desarrollan iniciativas para comprobar el uso o repercusión que tienen nuestras acciones en el 
entorno cercano desarrollando una intervención en el barrio del centro a fin de dar respuesta ante una de las problemáticas encontradas dentro del entorno natural de la zona, hecho en el que los protagonistas serán el alumnado al ser el propio artífice del cambio.

En las siguientes tabas se muestra el objetivo, actividades y relación de objetivos específicos de cada trimestre del proyecto "Seres solidarios con el medio ambiente".

Tabla 2

Propuesta de actividades primer trimestre proyecto "Seres solidarios con el medio ambiente"

PRIMER TRIMESTRE “¿Qué está pasando a nuestro mundo?”

\section{OBJETIVO}

Concienciación. Primera toma de contacto con el tema llevando un análisis de las propias practicas o exponiendo información desde diferentes soportes para que éstos sean conocedores de la situación.

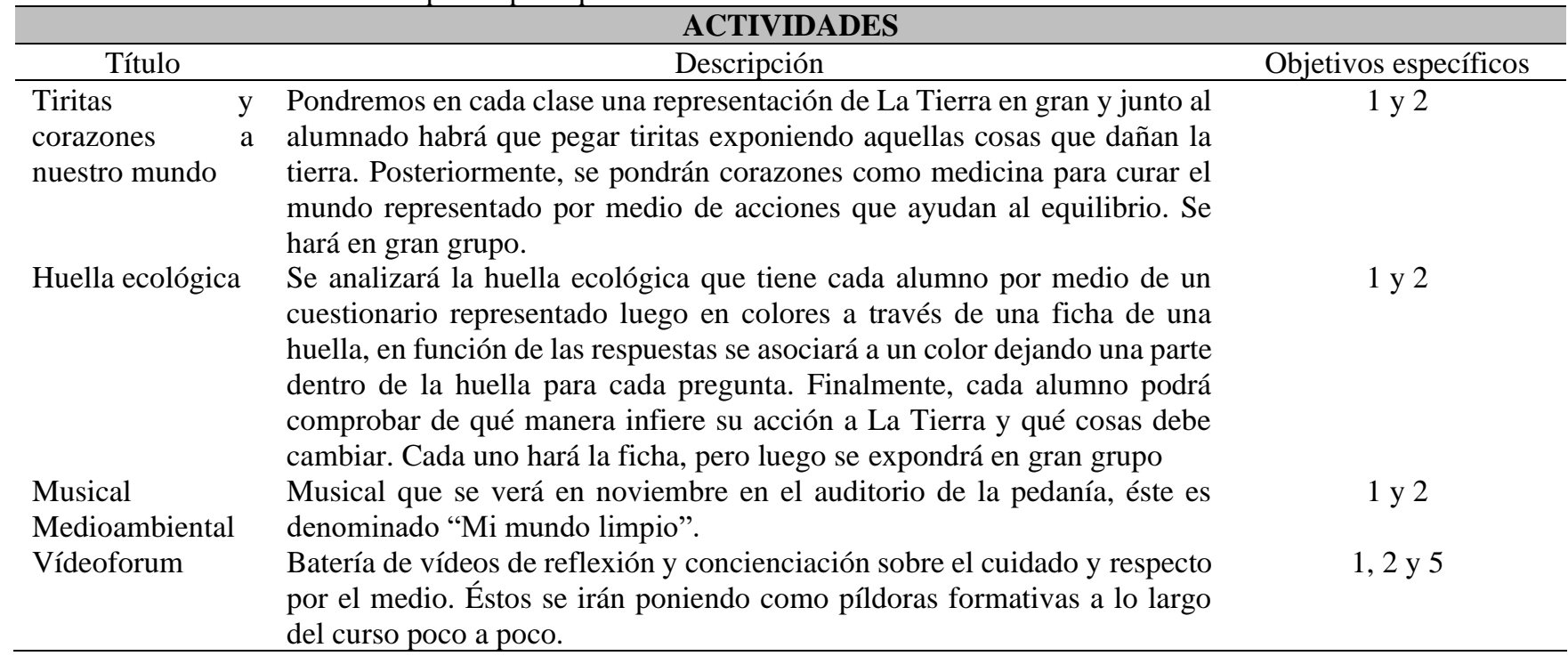

Tabla 3

Propuesta de actividades segundo trimestre proyecto "Seres solidarios con el medio ambiente" SEGUNDO TRIMESTRE "Manos a la obra" OBJETIVO

Experimentación. Toca abrir paso a la acción de pensar, un paso más allá del análisis. A partir de problemáticas deberán ser capaces de analizar y extraer ideas originales y creativas para hacer nuestro mundo un lugar mejor.

\section{ACTIVIDADES}

La caja medio ambiental: En cada clase habrá una caja en la cual cada quincena deberán tratar una situación práctica relacionada con un tópico medioambiental (agua, bosques, espacios, transportes, energías, reciclaje...) debiendo buscar en grupos una solución original al problema planteado. La mejor de cada clase en cada situación serán expuestas en el centro ante cada tópico siendo guardadas todas para ser expuestas en la exposición final.

\section{OBJETIVOS ESPECÍFICOS}

\section{$1,2,3$ у 5}


Tabla 4

Propuesta de actividades tercer trimestre proyecto "Seres solidarios con el medio ambiente"

TERCER TRIMESTRE "Somos ciudadanos eco-responsables"

\section{OBJETIVO}

Materialización. A partir de todo lo aprendido y experimentado, el alumnado deberá hacer visible todo lo aprendido por medio de la elaboración de creaciones propias que infiera en nuestro entorno y la población.

\begin{tabular}{|c|c|c|}
\hline \multicolumn{3}{|c|}{ ACTIVIDADES } \\
\hline Título & Descripción & Objetivos específicos \\
\hline Cartelería & $\begin{array}{l}\text { Elaboración de carteles en Plástica que ayude a la concienciación de la } \\
\text { población. }\end{array}$ & 1,2 y 6 \\
\hline Patrulla ecológica & $\begin{array}{l}\text { Grupo de alumnos que deberá llegar un rigor en el patio sobre la } \\
\text { manipulación de los residuos. Una manera de resaltar el protagonismo } \\
\text { infantil y hacer a nuestro alumnado responsable. }\end{array}$ & 2,3, y 5 \\
\hline Talleres & $1^{\circ}:$ Maracas (Botellas de actimel y arroz) & 3,5 y 6 \\
\hline materiales & $2^{\text {o: }}$ Señor de la cabeza de pasto & \\
\hline reciclados & $3^{\circ}$ Casas para pájaros (Brick de leche) & \\
\hline Exposición & $\begin{array}{l}\text { En un lugar del centro se expondrá todo lo elaborado a lo largo del curso } \\
\text { como modo de exposición. }\end{array}$ & - \\
\hline $\begin{array}{l}\text { Vídeo "Queridos } \\
\text { nuevos } \\
\text { ciudadanos..." }\end{array}$ & $\begin{array}{l}\text { En parejas de niños se hará un visionado explicando a los nuevos niños del } \\
\text { futuro cómo se han de comportar para hacer que nuestro mundo siga para } \\
\text { generaciones próximas. }\end{array}$ & $1,2,3$ у 4 . \\
\hline
\end{tabular}

\subsection{RECURSOS}

Para el cumplimiento de los propósitos pre-establecidos se construye una serie de recursos que sirven como apoyo para ser ejecutados en las actividades y llegar así, al acceso con éxito de los objetivos. Para su especificación vamos a dividir principalmente en dos grandes bloques: materiales y humanos. Los recursos materiales se dividen en didácticos o fungibles, los primeros son aquellos que resultan pieza clase dentro del entramado didáctico como medio facilitador y los segundos se corresponden con los que se utilizan como utensilios diarios en cualquiera de las actividades. Dentro de éstos se contemplan los siguientes recursos:

Tabla 5

Relación de recursos didácticos y no didácticos (fungibles)

\begin{tabular}{ll}
\multicolumn{1}{c}{ Didácticos } & \multicolumn{1}{c}{ No didácticos } \\
\hline Caja de resolución medio ambiental & Colores \\
Ficha didáctica de análisis del medio & Papelería de diversos materiales y tamaños \\
Mapamundi de situaciones medioambientales & Cartón \\
Ficha huella ecológica & Goma eva \\
Lista de enlaces de vídeo & Pegamento \\
Recursos resultantes de los materiales reciclados & Tijeras \\
& Camara de vídeo \\
& Bolígrafos. \\
\hline
\end{tabular}

Por otro lado, se aprecian también los seres humanos como un recurso más pues son los principales artífices del desarrollo de todo el entramado de materiales que se ponen a disposición del proyecto diseñado. Dentro de este tipo de recursos se contemplan: el profesorado implicado, el propio alumnado, las familias y el órgano de gobierno municipal. 


\subsection{EVALUACIÓN}

De cara a la evaluación de la intervención, en pro de asegurar el correcto funcionamiento y, por lo tanto, adquisición y cumplimiento de los objetivos planteados, se articulo un proceso sistemático de evaluación para cada uno de los objetivos planteados:

Tabla 6

Relación de objetivos específicos e instrumentos de evaluación

Objetivo específico
Concienciar sobre las
problemáticas que ahondan en la
interacción con nuestro medio
natural.

Instrumento de evaluación

Escala de observación: rúbrica con una serie de ítems que recoge todos los aspectos actitudinales y de valores al respecto de la temática medioambiental. Éstos podrán ser puntuados de acuerdo a una escala de 1 a 4 que se cumplimentarán a partir de la observación recogida a lo largo de las sesiones. Dado el volumen de actividades y la temporalidad que ocupa, nos apoyaremos en un registro anecdótico para cumplimentar la información.

Identificar las situaciones o Fichas o murales didácticas: A través de actividades dinámicas y elementos que generan cooperativas, éstos deberán reflejar un producto de aprendizaje que contaminación en nuestro muestra aquello que saben o no saben. Uno de los aspectos a extraer perjudicando las condiciones es el referente al siguiente objetivo específico. Algunos de ellos son: naturales del mismo.

Tomar un rol participativo y activo en la toma de decisiones e iniciativas en pro de la protección y cuidado del medio ambiente.

Redactar y elaborar un plan de trabajo para asegurar la creación de un espacio que contemple el reciclado y limpieza como premisas básicas cartel a partir del visionado de vídeos prácticos, el tratamiento de un caso concreto en el que se estudien las causas y consecuencias de cada uno de estos casos como el del Mar Menor.

Asambleas: Dentro de las asambleas realizadas en gran grupo, donde se comentan varias situaciones y experiencias, queda palpado, de primera mano, a partir de la vivencia del tú a tú, el saber de cada uno de nuestros alumnos. Información que nos servirá de complemento para el registro de observación.

Patrulla Ecológica - registro actitudinal (gomets): A partir de la intervención de cada uno en este grupo ecológico que se conformará para los recreos desde cada clase (un vestido de amarillo, otro de verde y un último de azul). De esta forma, gracias a la supervisión del profesorado, se le pondrá a cada alumno un punto positivo por cada vez que ejercite su rol de forma adecuada. Así se hará a lo largo del curso comprobando si cada uno de los participantes ha demostrado que sabe desarrollar acciones responsables y, además transmitírselas a los demás.

Evaluación del proyecto a nivel interno: Por medio de reuniones periódicas se fue redactando y revisando cada uno de los apartados que componía este proyecto de centro asegurando que se creaba en base a las premisas básicas pre-planteadas. Se tendrá en cuenta: adecuación a la edad, fomento a la creatividad, contemplación de una dimensión práctica y actitudinales, involucración de las familias, promoción de la educación medioambiental y involucración de la cooperación.

Demostrar una actitud Registro anecdótico y escala de observación: Al igual que en el participativa, respetuosa y activa ante las diferentes situaciones compartidas y cooperativas en pro del medio ambiente.

Impulsar el ámbito artístico y creativo en el diseño y preparación de materiales de diferente indole en relación al campo conceptual de la educación primer objetivo quedará recogida toda la información de relevancia, fruto de la observación, dentro de un registro anecdótico para luego poder plasmarlo en la escala de observación planteada.

Productos del aprendizaje: Se valorará a partir de los materiales elaborados en dos de las actividades planteadas: mural artístico elaborado con materiales reciclables y materiales artísticos y, por otro lado, los materiales elaborados en cada curso a partir de los materiales reciclables: botellas, vasos y briks de leche. ecológica. 


\section{CONCLUSIONES}

Dentro de este trabajo se ha tenido la intención de difundir el proceso de creación y organización de una propuesta dentro de la etapa de Educación Primaria sobre la educación ambiental, como medio de referencia e inspiración para otros profesionales e instituciones dentro del mundo educativo. La divulgación quiere evitar que únicamente se desarrollen estas acciones de forma puntual para conciar al respefto en la comunidad científica, social y educativo puesto que, realmente son efectivas y ejecuten el verdadero rol de arma para el cambio social cuando se articulan en numerosas medidas, proyectos o cambios curriculares, impulsando también el cambio de concepción didáctica de la educación ambiental dentro de los centros educativos.

En lo que respecta a la intervención, hay que resaltar los altos índices de aprendizaje a lo largo de la intervención. Hechos que pueden ser palpables mediante las fichas didácticas, murales y, sobre todo, en las entrevistas con los padres por medio de las cuales se puede constatar cómo va modificándose las prácticas del alumnado dentro de su rutina diaria, encontrando también una forma de participación, conocimiento e involucración de las familias. Otra de las consecuencias que se podrían apreciar sería un cambio en la dinámica respecto al cuidado del entorno y el medio ambiente dentro del núcleo escolar, apreciando más cuidado por los recursos que tenemos y una correcta acción en relación a éstos, hecho que se tuviera en cuenta en el profesorado y el alumnado de todo el centro, encontrando en el proyecto una identificación de este como una semilla la cual ha germinado su acción sobre todos los agentes sin distinción de roles.

Cabe resaltar también como positivo la envoltura lúdica y práctica que involucra la acción a lo largo de toda la intervención, saliéndose del libro de texto, posibilitando el aprendizaje desde otras perspectivas que se alejan del mero enfoque conservador. Hecho que promueve en el entusiasmo, interés y motivación por parte del alumnado a lo largo de la acción puesto que ven una oportunidad poder salir de la situación rutinaria accediendo sobre nuevos entornos de aprendizaje que dan pie a nuevos aprendizajes. Es por ello que, existe una necesidad de elaborar acciones comunes entre clases, niveles y etapas a fin de ampliar las fronteras didácticas demarcadas por los sistemas conservadores en la que los muros de las propias aulas marcaban los límites docentes. Así, la apertura hacia el barrio también supone una ventaja más puesto que se resalta el rol social de las escuelas dentro de los entornos cercanos, manifestando la mayor importancia y necesidad del ejercicio de su ciudadanía dentro de su entorno cercano, haciéndolo a él protagonista hacia el cambio.

Por último, como propuesta de mejora, mencionar que hay una falta de inclusión (BernárdezGómez, 2021) de uno de los principales agentes educativos dentro de la comunidad educativa: las familias. En este tipo de eje educativos se hace necesaria la implementación de un itinerario educativo en el que las 
familias estén adscritas para que se asegure con éxito la continuidad educativa pues desde el hogar se ha de educar en este tipo de aspectos educativos puesto que la relevancia de éstos recae en uso dentro del ámbito informal, hecho que se da principalmente junto a las familias las cuales, si no acompañan, interponen varias dificultades para asegurar el éxito de la intervención (Hernández y Álvarez, 2019). El último obstáculo que cabe destacar es alto número de trabas y obstáculos que interponen los organismos institucionales para el desarrollo de trabajos mutuos entre ambas partes a fin de transcender los muros escolares y llegar al barrio. Al margen de todas estas dificultades, por muy farragosas que sean, merece la pena la ejecución de éstos pues un gran ejemplo de cómo con una pequeña intervención se pueden conformar montañas que abren paso a un un nuevo panorama pedagógico más próximos a los principios pedagógicos del siglo XXI. 


\section{REFERENCIAS}

Alonso, Y., Núñez, M. R. y Hernández, A. (2015). Gestión del conocimiento universitario: rol del instructor de arte y promotor cultural en la cultura ambiental de la comunidad. Revista Universidad y Sociedad, 7 (3), 104-111.

Álvarez-Muñoz, J.S. y Hernández Prados, M. A. (2016). Percepción de los padres de los hábitos físicodeportivos, alimenticios y académicos del alumnado de Educación Primaria. Revista Electrónica Interuniversitaria de Formación del Profesorado, 19(3), 209-217.

Belmonte, M. L.; Bernárdez-Gómez, A. (2020). Respuesta social al estado de aislamiento por coronavirus, percepciones sobre educación. Revista Conhecimento Online, 3, 30-49. https://doi.org/10.25112/rco.v3i0.2326

Bernárdez Gómez, A.; Belmonte, M. L. (2020a). School dropout, determinants, educational policies and subsequent itineraries. Research, Society and Development, [S. 1.], 9 (10), p. e6849109234. DOI: 10.33448/rsd-v9i10.9234.

Bernárdez Gómez, A.; Belmonte, M. L. (2020b). Vulnerabilidad y exclusión educativa, una problemática postergada. Revista GETS, 3 (1), 79-94.

Bernárdez-Gómez, A. (2021). La investigación sobre trayectorias educativas. South Florida Journal of Development, 2(1), 42-53. https://doi.org/10.46932/sfjdv2n1-005

Calixto Flores, R. (2008). Representaciones sociales del medio ambiente. Perfiles educativos, 30(120), 33-62.

Campo, L. (2014). Aprendizaje servicio y educación superior Una rúbrica para evaluar la calidad de proyectos. Tesis doctoral inédita. Universidad de Barcelona

González Gaudiano, Édgar (1997). Educación ambiental: historia y conceptos a veinte años de Tbilisi, México: SITESA.

Hernández, P., Álvarez, M. y Aranda, A. (2017). El problema de deserción escolar en la produccción científica educativa. Revista Internacional de Ciencias Sociales y Humanidades, 1, 89-112. Recuperado de https://www.redalyc.org/pdf/654/65456040007.pdf

Hernández Prados, M. Ángeles ., y Álvarez Muñoz, J. S. . (2019). Family leisure and academic achievement. Perception of the families. ITALIAN JOURNAL OF EDUCATIONAL RESEARCH, (23), 86105. Recuperado de https://ojs.pensamultimedia.it/index.php/sird/article/view/3685

Iríbar Fernández, M. (2008) Modelo pedagógico para potenciar la educación ambiental de comunidades impactadas por el desarrollo de la industria turística. Tesis doctoral inédita. Universidad de Holguín. Novo, M. (1995). La educación ambiental. Madrid: Universitas.

Novo, M. (1998). La Educación Ambiental a distancia: su alcance y posibilidades. Revista iberoamericana de educación,16, 101-115.

Pardo Díaz, A. (1995). La educación ambiental como proyecto. Barcelona: ICE-Horsori.

Puig, J.M., Martín, X., y Batlle, R. (2008). Cómo iniciar un proyecto de aprendizaje y servicio solidario. Bilbao: Zerbikas. 
Puig, J. M., y Palos, J. (2006). Rasgos pedagógicos del aprendizaje-servicio. Cuadernos de Pedagogía, 357, 60-63.

Rubio, L. (2009). El aprendizaje en el aprendizaje servicio. En Puig, J.M. (coord.) Aprendizaje Servicio (ApS). Educación y compromiso cívico (pp.91-105). Barcelona: Graó.

Tapia, $\mathrm{M}^{\mathrm{a}}$ N. (2008). Aprendizaje y servicio solidario. Buenos Aires: Ciudad Nueva.

Trillo, F; Parada, A.; Bernárdez-Gómez, A. (2020). El impacto formativo de la enseñanza en la universidad: una experiencia de autoevaluación docente. Brazilian Journal of Development, 6(9). https://doi.org/10.34117/bjdv6n9-279

Vecina Jiménez, M. L., Chacón Fuertes, F., y Sueiro Abad, M. J. (2009). Satisfacción en el voluntariado: estructura interna y relación con la permanencia en las organizaciones. Psicothema, 21(1), 67-89. 